\title{
Star formation in the Small Magellanic Cloud: the youngest star clusters
}

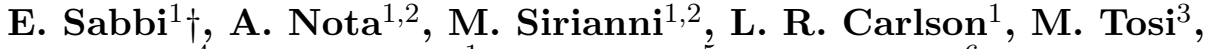 \\ J. Gallagher ${ }^{4}$, M. Meixner ${ }^{1}$, M. S. Oey ${ }^{5}$, A. Pasquali ${ }^{6}$, L. J. Smith ${ }^{7}$, \\ M. Vlajic ${ }^{8}$ and L. Hawks ${ }^{9}$ \\ ${ }^{1}$ STScI, 3700 San Martin Drive, Baltimore, MD, 21218, USA \\ email: sabbistsci.edu \\ ${ }^{2}$ ESA: Research \& Scientific Support Department; ${ }^{3}$ INAF-Osservatorio Astronomico di \\ Bologna, I; ${ }^{4}$ Dept. of Astronomy, University of Wisconsin, USA; ${ }^{5}$ University of Michigan, \\ USA; ${ }^{6}$ MPIA, Heidelberg, Germany; ${ }^{7}$ University College London, UK; ${ }^{8}$ University of Oxford, \\ UK; ${ }^{9}$ Rice University, USA
}

\begin{abstract}
We recently launched a comprehensive ground based (ESO/VLT/NTT) and space (HST \& SST) study of the present and past star formation in the Small Magellanic Cloud (SMC), in clusters and in the field, with the goal of understanding how star and cluster formation occur and propagate in an environment of low metallicity, with a gas and dust content that is significantly lower than in the Milky Way. In this paper, we present some preliminary results of the "young cluster" program, where we acquired deep F555W $(\sim \mathrm{V})$, and F814W $(\sim \mathrm{I}) \mathrm{HST} / \mathrm{ACS}$ images of the four young and massive SMC star clusters: NGC 346, NGC 602, NGC 299, and NGC 376.
\end{abstract}

Keywords. galaxies: individual (SMC), galaxies: star clusters, stars: pre-main-sequence, stars: formation, techniques: photometric

\section{Introduction}

The Small Magellanic Cloud (SMC) is an excellent laboratory to investigate the star formation (SF) processes and the associated chemical evolution in dwarf galaxies. Its current sub-solar chemical abundance $(Z=0.004)$ makes it the best local counterpart to the large majority of dwarf irregular (dIrr) and Blue Compact Dwarf (BCD) galaxies, whose characteristics may be similar to those in the primordial universe. The SMC proximity allows us to resolve into single stars the youngest and most compact star clusters, down to the sub solar mass regime.

Here we present some preliminary results of an in-depth study of the stellar content of the four youngest and most massive star clusters (NGC 346, NGC 602, NGC 299 and NGC 376) in the SMC. The clusters differ in age ( $~ 3$ to $30 \mathrm{Myr})$, regime of star formation, and location within the galaxy (see Fig. 1). For each cluster we obtained deep ACS/HST images in the F555W and F814W filters.

\section{The young clusters}

\section{1. $N G C 346$}

NGC 346 is an extremely young, ( $3 \mathrm{Myr}$, Bouret et al. 2003) moderately compact cluster, that excites the largest and brightest HII region - N66 - in the SMC (Relaño, Peimbert, \& Beckman 2002). NGC 346 lies in a very active and interesting region: at a

$\dagger$ Present address: STScI, 3700 San Martin Drive, Baltimore, MD, 21218, USA. 


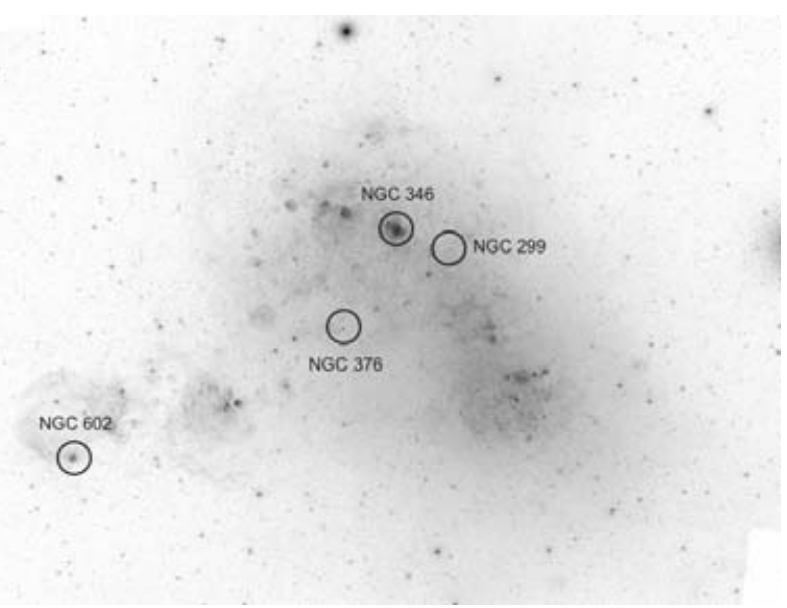

Figure 1. Position of the four clusters in the SMC.

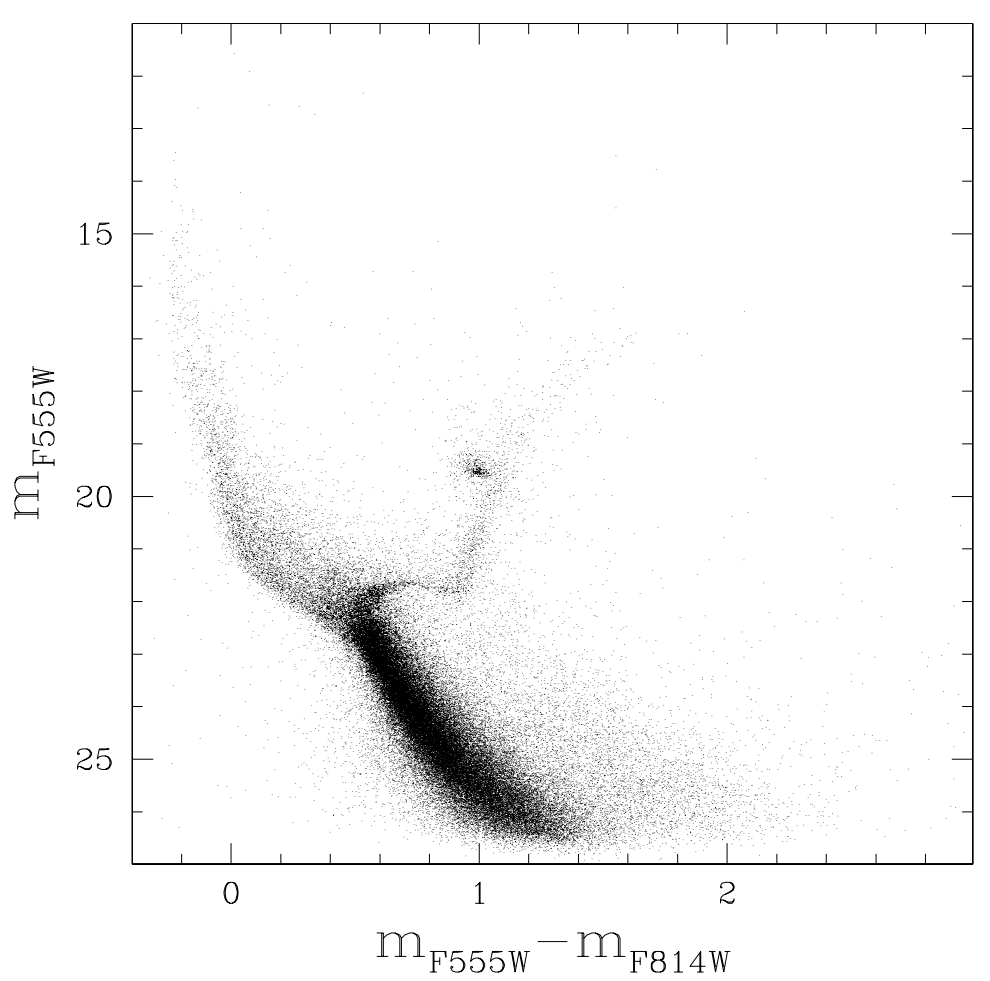

Figure 2. NGC 346 CMD $\left(m_{\mathrm{F} 555 \mathrm{~W}}\right.$ vs. $\left.m_{\mathrm{F} 555 \mathrm{~W}}-m_{\mathrm{F} 814}\right)$ for all stars with associated photometric errors smaller than 0.1 in both filters.

distance of 2 arc-minutes to the East (35 pc in projection) of the center of the cluster, we find the massive Luminous Blue Variable star HD 5980. N66 contains also at least two known supernova remnants (SNR): SNR 0057-7233 (Ye, Turtle, \& Kennicutt 1991), located to the Southwest of N66, and SNR 0056-7226.

In Figure 2 we show the $m_{\mathrm{F} 555 \mathrm{~W}}$ vs. $m_{\mathrm{F} 555 \mathrm{~W}}-m_{\mathrm{F} 814 \mathrm{~W}}$ Color-Magnitude Diagram $(\mathrm{CMD})$ of all the stars with photometric error $\sigma_{D A O}<0.1$ mag detected in the NGC 346 


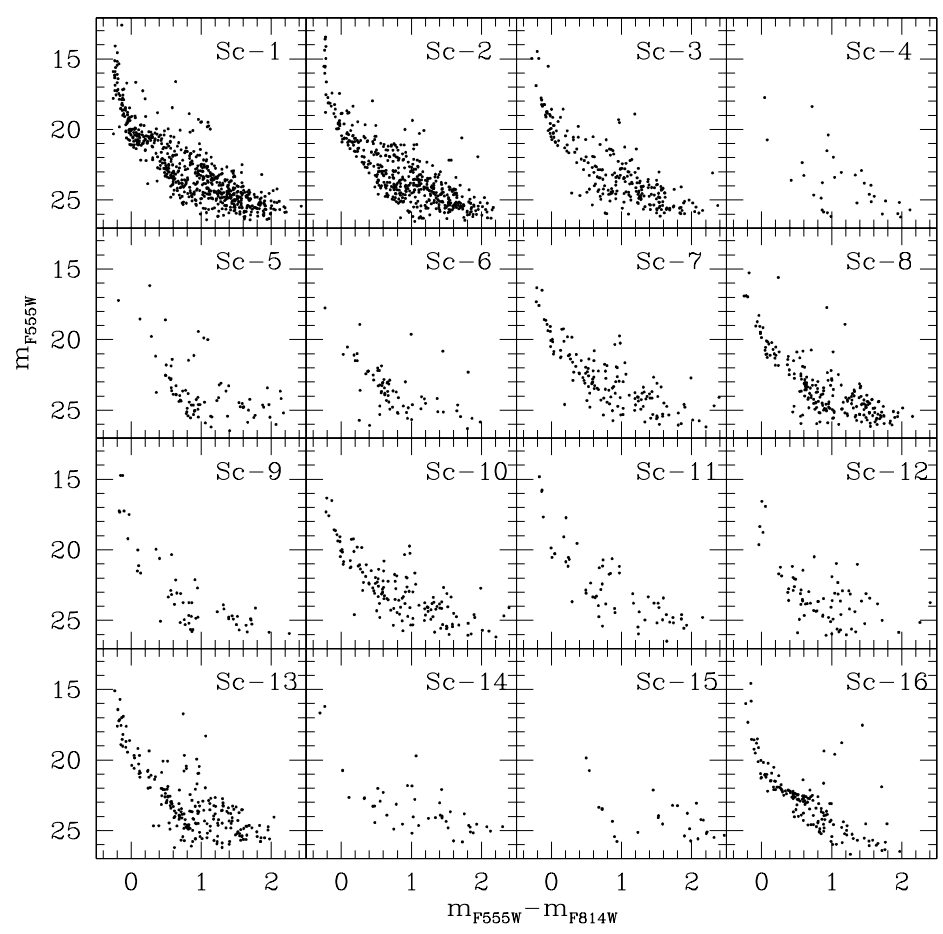

Figure 3. CMDs of the 16 sub-clusters identified within the NGC 346 region.

area. A first inspection of this CMD reveals that different stellar populations are present in the area:

- An old stellar population that belongs to the SMC field. The morphology of the CMD suggests that, in the field, a major episode of SF occurred approximately between 3 and 5 Gyr ago, but stars with ages up to at least 10 Gyr are also present. We also note that at most recent epochs the SF activity in the field has been significantly lower, with a possible moderate enhancement $\sim 150 \mathrm{Myr}$ ago.

- A young stellar population that belongs to NGC 346. A comparison with Padua isochrones indicates that NGC 346 is $3 \pm 1 \mathrm{Myr}$ old. The main sequence (MS) of the young population abruptly interrupts at $\mathrm{F} 555 \mathrm{~W} \simeq 21$. At $\mathrm{F} 555 \mathrm{~W}>21$ we identify hundreds of red and faint stars whose colors and magnitudes are consistent with those predicted for low mass $\left(0.6-3 \mathrm{M}_{\odot}\right)$ pre-Main Sequence (pre-MS) stars (see also Nota et al. 2006), that likely formed at the same time of the central cluster.

The high spatial resolution of our observations shows that the youngest stellar population is not uniformly distributed within the ionized nebula: we identify at least 15 sub-clusters, which differ in size and stellar content (see Fig. 3). Within the uncertainties of the comparison with isochrones, the sub-clusters are likely coeval with each other. However, we also find a relatively older sub-cluster ( $\mathrm{Sc}-16$, with an age $15 \pm 2.5 \mathrm{Myr}$ ), located at the Northeast periphery of our data. This sub-cluster is likely not related to the star forming episode that originated NGC 346 (see also Sabbi et al. 2007). 

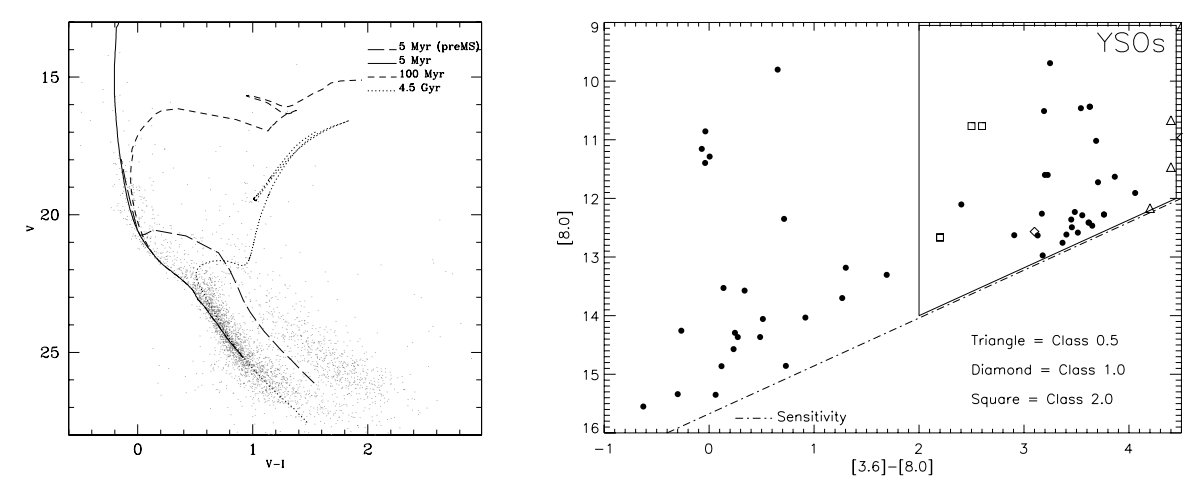

Figure 4. Left Panel: NGC 602 CMD ( $m_{\mathrm{F} 555 \mathrm{~W}}$ vs. $\left.m_{\mathrm{F} 555 \mathrm{~W}}-m_{\mathrm{F} 814}\right)$. Padua isochrones have been overlayed to estimate the age of the different stellar populations. Right Panel: NGC 602 IR CMD $([8.0 \mu],[3.6 \mu]-[8.0 \mu])$. The expected YSO location is indicated by the box, derived from stellar models (Whitney et al. 2004)

\subsection{NGC 602}

Very little is known about the young cluster NGC 602, located in a low stellar density region, the "Bridge" of the SMC, stretching between Shapley's wing (1940) and the main body of the SMC.

Figure 4 (left panel) shows the $m_{\mathrm{F} 555 \mathrm{~W}}$ vs. $m_{\mathrm{F} 555 \mathrm{~W}}-m_{\mathrm{F} 814 \mathrm{~W}} \mathrm{CMD}$ of NGC 602 . The CMD reveals a rich population of pre-MS low mass $\left(0.6-3 \mathrm{M}_{\odot}\right)$ stars, likely formed coevally with the cluster $\sim 5$ Myr. In the field, a major episode of SF occurred approximately $\sim 6$ Gyr ago, and a second, moderate, episode occurred $\sim 150 \mathrm{Myr}$ ago.

SST data (see Fig. 4-right panel) reveals the presence of a possibly younger generation of young stellar objects (YSOs likely of class 0.5, and I), still embedded in their dust cocoons, likely younger than $1 \mathrm{Myr}$. We infer that the powerful winds developed by the most massive $\mathrm{O}$ stars in the center of the cluster are triggering new episodes of star formation in the periphery, where the dust concentration is higher (Carlson et al. 2007, in prep.).

\section{3. $N G C 299 \& 3 G C 376$}

NGC 299, located in the main body of the SMC, and NGC 376, in the "Wing" of the galaxy, are the oldest star clusters in our survey $(\sim 25 \mathrm{Myr})$. In these clusters the winds from the most massive stars have already dispersed all the gas and dust, and star formation is no longer active (see Fig 5). In these two regions, the field of the SMC had a major episode of SF approximately between 4 and 6 Gyr ago, while at most recent epochs the SF activity has been significantly lower, with a possible enhancement $\sim 150$ Myr ago.

It is known that massive star winds at low metallicity are less powerful and efficient. The observations of these two "older clusters" provide an interesting measure of the efficiency of the feedback mechanism at low metallicity. In addition, the clusters show very similar physical characteristics in spite of the very different local environment conditions, in terms of stellar and gas/dust density, suggesting that local condition may have little impact on cluster formation and evolution.

\section{Conclusions}

Our observation allowed us to follow in detail the evolution of SMC star clusters during their first $30 \mathrm{Myr}$ of lifetime. Our results indicate that star formation is still active in 

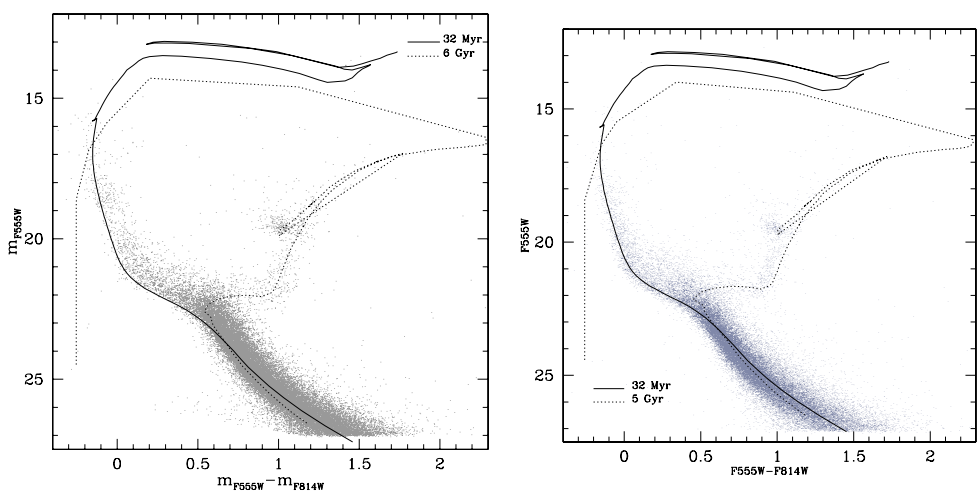

Figure 5. Left Panel: NGC 299 CMD ( $m_{\mathrm{F} 555 \mathrm{~W}}$ vs. $\left.m_{\mathrm{F} 555 \mathrm{~W}}-m_{\mathrm{F} 814}\right)$. Right Panel: NGC 376 CMD $\left(m_{\mathrm{F} 555 \mathrm{~W}}\right.$ vs. $\left.m_{\mathrm{F} 555 \mathrm{~W}}-m_{\mathrm{F} 814}\right)$. Padua isochrones have been overlayed to estimate the age of the different stellar populations.

the SMC, and that metallicity and local conditions do not seem to affect star and cluster formation processes.

Our survey provides a snapshot of the star formation history of the SMC over a lookback time of about $10 \mathrm{Gyr}$. In all observed fields we can distinguish a major episode of star formation, occurred approximately between 3 and 6 Gyr ago, but stars with ages up to 10 Gyr are also present. We note also that at most recent epochs the star formation activity in the field has been significantly lower, with a possible moderate enhancement 150 Myr ago.

\section{References}

Bouret, J.C., Lanz, T., Hillier, D.J., Heap, S.R., Hubeny, I. \& Lennon, D. J. 2003, ApJ 595, 1182

Hilditch, R.W., Howarth, I.D. \& Harries, T.J. 2005, MNRAS 125, 336

Nota, A., Sirianni, M., Sabbi, E., Tosi, M., Meixner, M., Gallagher, J., Clampin, M., Oey, S., Smith, L.J., Walterbos, R. \& Mack, J. 2006, ApJ 640, L29

Relaño, M., Peimbert, M. \& Beckman, J. 2002, ApJ 564, 704

Rubio, M., Contursi, A., Lequeux, J., Probst, R., Barbà, R.H., Boulanger, F., Cesarsky, D. \& Maoli, R. 2000, A\&SA 359, 1139

Sabbi, E., Sirianni, M., Nota, A., Tosi, M., Gallagher, J., Meixner, M., Oey, M.S., Walterbos, R., Pasquali, A., Smith, L.J. \& Angeretti, L. 2007, $A J$ in press (astro-ph/0609330)

Walborn, N.R. \& Parker, J.W. 1992, ApJ 399, L87

Walborn, N.R., Maíz-Apellániz, J. \& Barbá, R.H. 2002, AJ 124, 1601

Whitney, B.A., Indebetouw, R., Bjorkman, J.E. \& Wood, K. 2004, ApJ 617, 1177

Ye, T., Turtle, A.J. \& Kennicutt, R.C., Jr. 1991, MNRAS 249, 722

\section{Discussion}

BRANDL: You mentioned an older cluster to the northwest of NGC 346. Can you provide an age estimate for that cluster?

SABBI: Yes, we computed a synthetic CMD for the old cluster. In doing this we derive an age of $4.3 \pm 0.1$ Gyr.

BLITZ: Is there any remnant molecular cloud within 50-100 pc of NGC 299 or NGC $376 ?$

SABBI: There are not molecular clouds close to these 2 clusters. 